\title{
Ultraviolet nanoimprinted polymer nanostructure for organic light emitting diode application
}

\author{
Sohee Jeon, ${ }^{1}$ Jae-Wook Kang, ${ }^{1, a)}$ Hyung-Dol Park, ${ }^{1}$ Jang-Joo Kim, ${ }^{1, b)}$ Jae R. Youn, ${ }^{1, b)}$ \\ Jongyoup Shim, ${ }^{2}$ Jun-ho Jeong, ${ }^{2}$ Dae-Geun Choi, ${ }^{2}$ Ki-Don Kim, ${ }^{2}$ Ali Ozhan Altun, ${ }^{2}$ \\ Se-Heon Kim, ${ }^{3}$ and Yong-Hee Lee ${ }^{3}$ \\ ${ }^{1}$ Department of Materials Science and Engineering, Seoul National University, Seoul 151-744, \\ Republic of Korea \\ ${ }^{2}$ Nano-Mechanical Systems Research Center, Korea Institute of Machinery and Materials, Daejeon 305-343, \\ Republic of Korea \\ ${ }^{3}$ Department of Physics, Korea Advanced Institute of Science and Technology, Daejeon 305-701, \\ Republic of Korea
}

(Received 22 April 2008; accepted 14 May 2008; published online 5 June 2008)

\begin{abstract}
Light extraction efficiency of a conventional organic light emitting diode (OLED) remains limited to approximately $20 \%$ as most of the emission is trapped in the waveguide and glass modes. An etchless simple method was developed to fabricate two-dimensional nanostructures on glass substrate directly by using ultraviolet (UV) curable polymer resin and UV nanoimprint lithography in order to improve output coupling efficiency of OLEDs. The enhancement of the light extraction was predicted by the three-dimensional finite difference time domain method. OLEDs integrated on nanoimprinted substrates enhanced electroluminance intensity by up to $50 \%$ compared to the conventional device. (C) 2008 American Institute of Physics. [DOI: 10.1063/1.2939554]
\end{abstract}

Light extraction efficiency of a normal bottom emission organic light emitting diode (OLED) is limited to approximately $20 \%$ and $80 \%$ of the emitted light is trapped in the waveguide and glass modes. ${ }^{1,2}$ Light extraction from OLEDs becomes the most serious factor limiting the overall efficiency of OLEDs for displays and solid state lighting. There have been many efforts to improve the output coupling efficiency of OLEDs by modifying the substrate surface. ${ }^{3-9}$ Recently, 2D slab photonic crystal (PC) structures had been used and the output coupling efficiency was enhanced by more than $50 \% .^{10-12}$ The nanoimprint lithogrophy (NIL) process is an attractive method to replace the expensive photolithography and etching process for fabrication of nanoscale PCs. ${ }^{13}$ It has been applied to direct fabrication of a PC structure in a glass substrate by using a so called "hot embossing" technique, where high pressure of about 20 bars and high temperature of about $300{ }^{\circ} \mathrm{C}$ are required. ${ }^{12} \mathrm{~A}$ simple and cost-effective PC patterning method is still needed to achieve high throughput in the atmosphere.

In this study, an etchless UV-NIL process is examined for patterning of an organic PC structure onto an OLED substrate under atmospheric pressure and at room temperature. The UV-NIL process for the direct patterning of polymer pillars is depicted in Fig. 1. Surface of the stamp was coated by a self-assembled monolayer of trichloro- $(1 \mathrm{H}, 1 \mathrm{H}, 2 \mathrm{H}, 2 \mathrm{H}$-perfluorooctyl) silane for easy separation. $60 \mu \mathrm{l}$ of a UV curable resin was dispensed onto the Si stamp and a glass wafer was placed on it and finally cured by UV exposure for 3 min with an illumination power of $14.4 \mathrm{~mW} \mathrm{~cm}^{-2}$. The UV curable resin is composed of two monomers, tri(propylene glycol) diacrylate (Alddrich) and tri(methylol) propane triacrylate (Aldrich). Subsequently,

\footnotetext{
${ }^{\text {a) }}$ Present address: Department of Surface Technology Korea Institute of Materials Science (KIMS) Changwon, 641-831 (Korea).

${ }^{b)}$ Authors to whom correspondence should be addressed. Electronic addresses: jjkim@snu.ac.kr and jaeryoun@snu.ac.kr.
}

photoinitiator of 2,2-dimethoxy-2-phenylacetophenone (Aldrich) was added to the monomer mixture at $4 \mathrm{wt} \%$. Rad $2200 \mathrm{~N}$ (TEGO Chemi Service) of $10 \mathrm{wt} \%$ was blended as a releasing agent with the prepared mixture. Deposition of a $\mathrm{SiN}_{x}$ layer on the polymer pillar array as the buffer layer completes the formation of PC structure on the glass substrates. The $\mathrm{SiN}_{x}$ layer is transparent and has high refractive index ( 2.02). It was deposited to make the PC surface planar and to give higher refractive index than that of polymer pillars. In order to achieve a flat surface, $\mathrm{SiN}_{x}$ is deposited on the PC layer and two types of PCs with different $\mathrm{SiN}_{x}$ layer thicknesses $(500$ and $800 \mathrm{~nm})$ were prepared.

The 8 in. silicon wafer was used as the stamp materials after patterned by the deep UV lithography and reactive ion
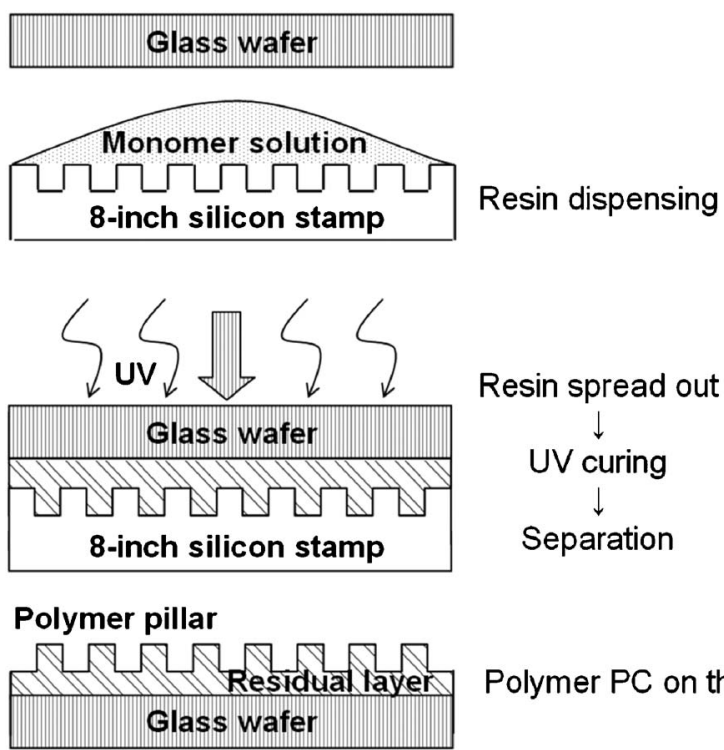

Polymer PC on the wafer

FIG. 1. Schematic of the UV-NIL procedure for the direct fabrication of a polymer pillar substrate. 


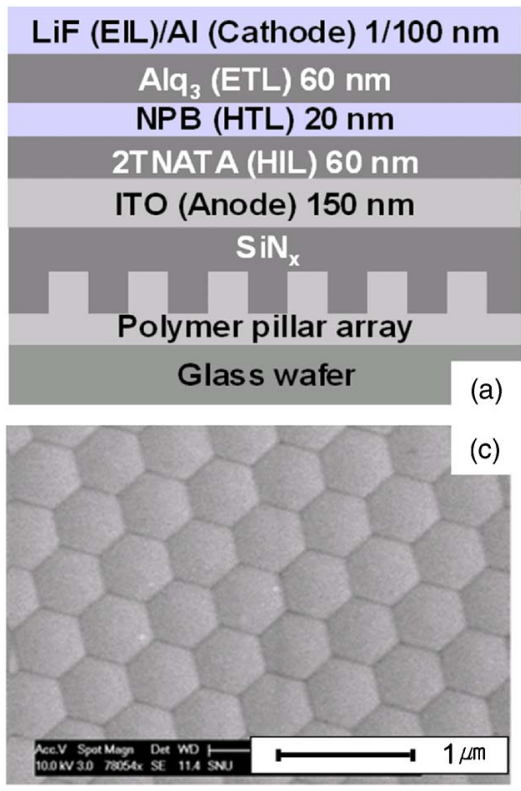

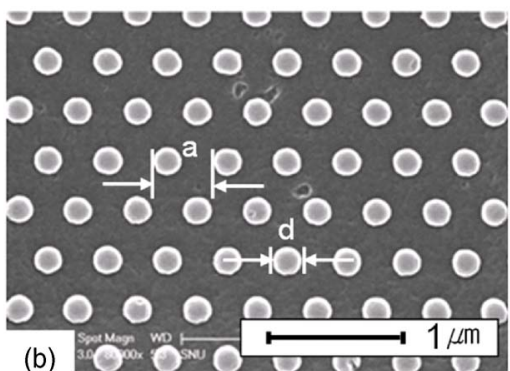

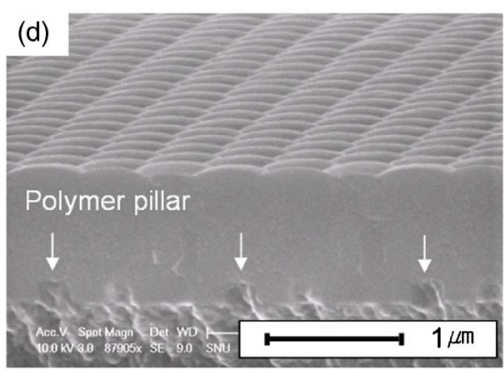

FIG. 2. (Color online) (a) Vertical structure and materials of OLEDs with PC structure in the substrate. (b) SEM image of the imprinted polymer pillars. (c) Top view of the PC slab. (d) Cross-sectional view of the PC slab. etching. The silicon stamp pattern has a hexagonal lattice structure with repeated circular holes. The lattice constant of the holes is $530 \mathrm{~nm}$ and both the diameter and the height are $265 \mathrm{~nm}$, which will produce the polymer pillar whose aspect ratio is $1: 1$. All propagating visible-wavelength light is in the nonguided mode region at that lattice constant and above the band gap of photonic band structure. Hence, greater light intensity can be achieved at oblique viewing angles in the visible region. ${ }^{10}$ Figure 2 (b) shows the scanning electron microscope (SEM) image of the fabricated PC. $\mathrm{SiN}_{x}$ completely fills the gap between the polymer pillars without voids and the surface becomes smoother by depositing the buffer layer [Figs. 2(c) and 2(d)].

In order to predict the performance of the proposed device, a three-dimensional finite difference time domain (FDTD) simulation was performed. ${ }^{14}$ The simulation structure included air, a glass substrate, a polymer resin, $\mathrm{SiN}_{x}$, indium tin oxide, organic layers, and cathode electrode layers. The polymeric PC was modeled using a circular rod shape and the measured geometric parameters. The simulation domain size was $3.13 \times 2.71 \times 6.19 \mu \mathrm{m}^{3}$ and the time step was 0.0333 fs. The periodic boundary conditions were used on the four vertical sides of the simulation domain in order to reduce the simulation time, and the array size and the two upper and lower horizontal sides were set to have perfectly matched layer boundary conditions. To model the cathode aluminum electrode, the auxiliary differential equations method was employed where the single-pole Drude model was assumed. ${ }^{14}$ Many Gaussian dipole sources were randomly distributed over the emission layer and the initial phase and the direction of dipole oscillation were randomly assigned at the beginning of the simulation. The center frequency of each Gaussian dipole source was set to be the value corresponding to the OLED peak emission wavelength of $511 \mathrm{~nm}$ and the time duration of the Gaussian pulse was determined on the order of femtosecond for the source to have a broad emission wavelength band of about $50 \mathrm{~nm}$. The simulation result is shown in Fig. 3. The $y$ axis is the time integration of the Poynting vector in the $z$ direction over the top surface in air. The integrated $z$-Poynting vector is a measure of the light emission out of the glass into the air. The extraction efficiency remained nearly constant for various

Downloaded 30 Jun 2008 to 147.46 .94 .95 . Redistribution subject glass thickness, as shown in Fig. 3. Therefore, the air layer can be included in calculation structure without excessive time consumption. The predicted light extraction efficiency of the polymer PC was enhanced by nearly $70 \%$.

Enhancement of the light extraction efficiency in the forward direction (glass side) and emission characteristics are also confirmed from the experiments. Relative integrated spectra of the light emitted from the device were measured using an integrating sphere (Labsphere Co., 6 in. diameter). Figure 4 shows the emission spectra of the device with relative EL intensities. The electroluminance (EL) intensity of the PC device increases by more than $50 \%$ compared to the conventional device without a PC. Moreover, the enhancement of the light extraction from the PC-OLEDs is represented by means of the integrated light-output power versus the current density, as shown in Fig. 5. The EL intensity was increased by $54 \%$ compared to the conventional device. Thinner $\operatorname{SiN}_{x}$ buffer layer gives a little higher light extraction

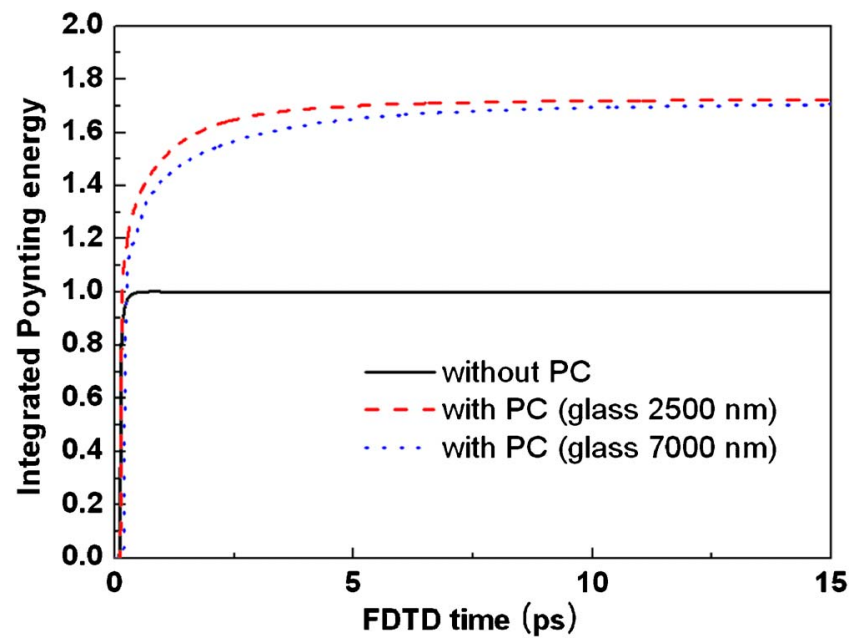

FIG. 3. (Color online) FDTD simulation results with variation in the glass thickness. The enhancement of the light extraction is nearly constant, with reasonable thickness variation. The simulation shows no thickness effect; therefore, adopting the glass thickness in the calculation is shown to be feasible. The enhancement is nearly $70 \%$ compared to a conventional

OLED.
AIP license or copyright; see http://apl.aip.org/apl/copyright.jsp 


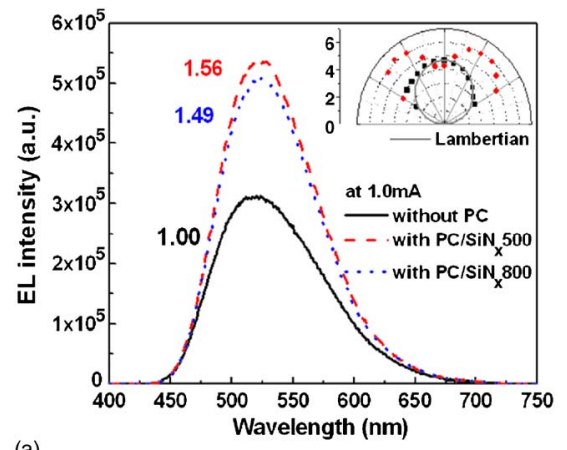

(a)

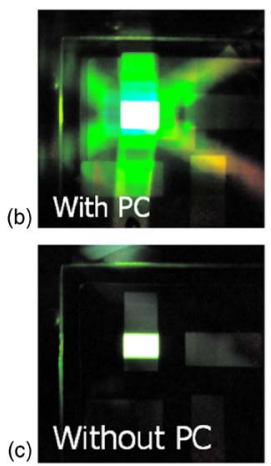

FIG. 4. (Color online) (a) EL intensity using integrating sphere measurement, Inset: radiation profile represented in terms of luminance efficiency $\left(\mathrm{cd} \mathrm{A}^{-1}\right)$. Photographs (b) with and (c) without the PC-OLEDs.

efficiency because the thick $\mathrm{SiN}_{x}$ film causes additional light absorption.

As shown in the emission images in Fig. 4, the conventional OLED emits light with a symmetric distribution in all in-plain directions, while the PC device produces a pattern with sixfold symmetry, reflecting the hexagonal structure of the PC layer. Moreover, the profiles are represented in terms of the luminance efficiency in the inset of Fig. 4. The maximum luminance efficiency of the PC device is $5.95 \mathrm{~cd} \mathrm{~A}^{-1}$ at a tilted direction of $40^{\circ}$, which is an improvement of nearly
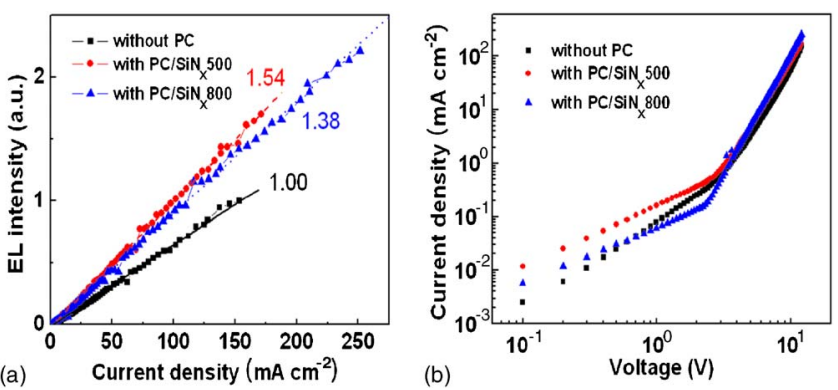

FIG. 5. (Color online) (a) The light-output power from the OLEDs with and without the $\mathrm{PC}$, (b) $J-V$ characteristics.
$30 \%$ of the maximum luminance of the reference. The profile of the reference device is very similar to the Lambertian profile, whereas the $\mathrm{PC}$ device produces a radiation shape that is similar to butterfly wings whose area is about 1.5 times larger than the reference. The OLEDs integrated on PC structures not only give high luminance efficiency but also exhibit interesting emission patterns depending on the symmetry and dimensions of the pillars such as lattice constant, diameter, height, and arrangement. ${ }^{15,16}$ In conclusion, an etchless UV-NIL process is developed to fabricate organic PC structures as an OLED substrate with 50\% enhancement of the device efficiency.

${ }^{1}$ C. F. Madigan, M.-H. Lu, and J. C. Strum, Appl. Phys. Lett. 76, 1650 (2000).

${ }^{2}$ N. C. Greenham, R. H. Friend, and D. D. C. Bradley, Adv. Mater. (Weinheim, Ger.) 6, 491 (1994).

${ }^{3}$ I. Schnitzer and E. Yablonovitch, Appl. Phys. Lett. 63, 2174 (1993).

${ }^{4}$ S. Moller and S. R. Forrest, J. Appl. Phys. 91, 3324 (2002).

${ }^{5}$ T. Tsutsui, M. Yahiro, H. Yokogawa, K. Kawano, and M. Yokoyama, Adv. Mater. (Weinheim, Ger.) 13, 1149 (2001).

${ }^{6}$ M. Agrawal, Y. Sun, S. R. Forrest, and P. Peumans, Appl. Phys. Lett. 90, 241112 (2007).

${ }^{7}$ J. M. Ziebarth and M. D. Mcgehee, J. Appl. Phys. 97, 064502 (2005).

${ }^{8}$ B. J. Matterson, J. M. Lupton, A. F. Safonov, M. G. Salt, W. L. Barnes, and I. D. W. Samuel, Adv. Mater. (Weinheim, Ger.) 13, 123 (2001).

${ }^{9}$ H. J. Peng, Y. L. Ho, X. J. Yu, and H. S. Kwok, J. Appl. Phys. 96, 1649 (2004).

${ }^{10}$ S. Fan, P. R. Villeneuve, J. D. Joannopoulos, and E. F. Schubert, Phys. Rev. Lett. 78, 3294 (1997).

${ }^{11}$ Y. R. Do, Y.-C. Kim, Y.-W. Song, C.-O. Cho, H. Jeon, Y.-J. Lee, S.-H. Kim, and Y.-H. Lee, Adv. Mater. (Weinheim, Ger.) 15, 1214 (2003).

${ }^{12}$ K. Ishihara, M. Fujita, I. Matsubara, T. Asano, S. Noda, H. Ohata, A. Hirasawa, H. Nakada, and N. Shimoji, Appl. Phys. Lett. 90, 111114 (2007).

${ }^{13}$ S. Y. Chou, P. R. Krauss, and P. J. Renstrom, J. Vac. Sci. Technol. B 14, 4129 (1996).

${ }^{14}$ A. Taflove and S. C. Hagness, Computational Electrodynamics: The Finite-Difference Time-Domain Method, 2nd ed. (Artech House, Norwood, 2000).

${ }^{15}$ K. Sakoda, Optical Properties of Photonic Crystals (Springer, Berlin, 2001).

${ }^{16}$ S. G. Johnson and J. D. Joannopoulos, Photonic Crystals (Kluwer Academic, Boston, 2002). 